\title{
Lista das espécies de Phoridae (Insecta, Diptera) do Estado do Mato Grosso do Sul
}

\author{
Danilo César Ament
}

Universidade de São Paulo, Faculdade de Filosofia, Ciências e Letras de Ribeirão Preto, Departamento de Biologia, Av. Bandeirantes 3900, 14040-901, Ribeirão Preto, SP, Brasil. (danament@gmail.com)

ABSTRACT. Checklist of the Phoridae species (Insecta, Diptera) from the State of Mato Grosso do Sul. Records of seven species belonging to six genera and two subfamilies were found for the Mato Grosso do Sul state, Brazil. This number of species is far from being representative of the real fauna of the state and is much smaller than the number of known species of other better studied states.

KEYWORDS. Biodiversity, inventory, phorids, Biota-MS Program.

RESUMO. Registros de sete espécies de forídeos pertencentes a seis gêneros e duas subfamílias foram encontrados para o estado do Mato Grosso do Sul, Brasil. Esse número de espécies está longe de ser representativo da fauna real do estado, sendo muito aquém do número de espécies conhecidas de Phoridae para outros estados mais bem estudados.

PALAVRAS-CHAVE. Biodiversidade, inventário, forídeos, Programa Biota-MS.

A família Phoridae compreende pequenos dípteros de 0,4-7 mm de comprimento, característicos por sua venação alar reduzida, escudo curvado e por sua locomoção rápida e intermitente, à qual faz referência seu nome popular em inglês, scuttle flies. Os forídeos destacam-se por sua enorme diversidade morfológica e ecológica tanto de imaturos como de adultos. Sua diversidade larval é considerada uma das mais ricas em insetos, podendo ser predadores, parasitas, parasitoides, herbívoros, decompositores, polinizadores, fungívoros e onívoros (DisNey, 1994).

Atualmente existem cerca de 4.000 espécies descritas distribuídas em 250 gêneros. Estima-se que a fauna real da família, no entanto, possa ser até dez vezes maior em regiões menos estudadas, como a Região Neotropical (BROWN, 2004a). Só existem duas propostas de divisões de Phoridae em subfamílias (SCHMitz, 1929; Brown, 1992), sendo a de Brown (1992) a única embasada no método filogenético. Apesar dos grandes avanços na interpretação de caracteres e no relacionamento de grupos trazidos por essa última proposta, estudos recentes têm apontado para hipóteses de relacionamento bastante distintas e a necessidade de redelimitação das subfamílias.

Os sistematas do grupo no mundo sempre foram poucos para a dimensão da família. Trabalhos que abordem a fauna de forídeos do Brasil tratam principalmente da fauna do sul e sudeste do Brasil, a maioria deles de autoria do Frei Thomas Borgmeier, um dos principais especialistas mundiais de Phoridae.

\section{MATERIAL E MÉTODOS}

Para a elaboração desta lista de espécies foi consultado o catálogo mundial de Phoridae (BorGMEIER, 1968), os seus complementos (BORGMEIER, 1971; BROwn, 2012) e a versão online do Zoological Records (http://apps.webofknowledge. com).

\section{RESULTADOS E DISCUSSĀO}

Foram encontrados registros de sete espécies no Mato Grosso do Sul, pertencentes a duas subfamílias e seis gêneros distintos. Isso é claramente uma amostra pouco representativa da fauna do estado, provavelmente em consequência da falta de coletas e preparação de coleções. Como comparação, são exibidos a seguir os registros de forídeos das localidades onde a família é mais bem conhecida no Brasil: o estado do Rio de Janeiro, onde morou o frei Borgmeier, e o estado de Santa Catarina, em especial a localidade de Nova Teutônia, intensamente amostrada por Fritz Plaumann (Tab. I). 
Tab. I. Comparação do número de forídeos conhecidos para o estado do Mato Grosso do Sul e estados e localidade mais bem estudados do Brasil.

\begin{tabular}{|c|c|c|c|c|}
\hline & Mato Grosso do Sul & Santa Catarina & Rio de Janeiro & Nova Teutônia (SC) \\
\hline Espécies & 7 & 296 & 239 & 235 \\
\hline Gêneros & 6 & 47 & 57 & 35 \\
\hline Subfamílias & 2 & 4 & 4 & 2 \\
\hline
\end{tabular}

Lista das espécies de Phoridae do Mato Grosso do Sul

PHORINAE

Coniceromyia Borgmeier, 1923

piricornis Borgmeier, 1950. Localidade-tipo: Brasil, Mato

Grosso do Sul: Salobra. Refs.: BorgmeIER, 1963:451.

\section{METOPININAE}

Ecituncula (Labiduncula) Borgmeier, 1936

inquirenda (Silvestri, 1947). Localidade-tipo: Brasil, Mato

Grosso do Sul: Campo Grande.

\section{Ectochaeta Borgmeier, 1958}

spinulosa Borgmeier, 1958. Localidade-tipo: Brasil, Mato

Grosso do Sul: Caraguatá.

\section{Megaselia (Megaselia) Schmitz, 1927}

scalaris (Loew, 1866). Localidade-tipo: Cuba. Distribuição cosmopolita.

\section{Melaloncha Brues, 1904}

deinocerca Borgmeier, 1960. Localidade-tipo: Brasil, Mato

Grosso?: Fronteira oeste. Refs.: BRown, 2004b:28.

nigricorpus Borgmeier, 1934. Localidade-tipo: Bolívia,

Mapiri: Sarampioni (S. Carlos). Distribuição: Bolívia e

Brasil, Mato Grosso do Sul: Maracajú. Refs. BorgMEIER,
1959:176; BARNES, 1991; BROWN, 2004b:32.

\section{Pseudacteon Coquillett, 1907}

lontrae Mattos \& Orr, 2002. Localidade-tipo: Brasil, Mato Grosso do Sul: próximo à Passo do Lontra. Refs.: ORR et al., 2003.

Agradecimentos. A Fundação de Apoio ao Desenvolvimento do Ensino, Ciências e Tecnologia do Estado de Mato Grosso do Sul (Fundect) e a Superintendência de Ciências e Tecnologia do Estado de Mato Grosso do Sul (Sucitec/MS) pelo convite de participação neste fascículo especial da Iheringia, Série Zoologia e o suporte financeiro para sua publicação.

\section{REFERÊNCIAS BIBLIOGRÁFICAS}

BARNES, J. K. 1991. Additions to the phorid fauna (Diptera, Phoridae) of North America North of Mexico. Florida Entomologist 74:305-310.

Brown, B. V. 1992. Generic revision of the Phoridae of the Neartic Region and phylogenetic classification of Phoridae, Sciadoceridae, and Ironomyiidae (Diptera: Phoridea). Memoirs of the Entomological Society of Canada 164:1-114.

Brown, B. V. 2004. Diversity of ant-decapitating flies (Diptera: Phoridae: Apocephalus) from the ALAS Project: New results and projections. Sociobiology 44(3):683-688.

Brown, B. V. 2012. Update of Borgmeier's 1968 catalog of world phorids. Disponível em: <http://www.phorid.net/phoridae/phorcat. html>. Acessado em 27.08.2012.

Disney, R. H. L. 1994. Scuttle flies. The Phoridae. London, Chapman \& Hall. 467p.

ORR, M. R.; DAhLSTEN, D. L. \& Benson, W. W. 2003. Ecological interactions among ants in the genus Linepithema, their phorid parasitoids, and ant competitors. Ecology Entomology 28:203-210.

Schмiтz, H. 1929. Revision der Phoriden. Berlin, Ferd Dummlers. 211p. 